\title{
Focus in Honor of Yinsheng Wang, Recipient of the 2013 Biemann Medal
}

$\mathrm{N}$ o matter how apparently important a personal accomplishment in science, few pleasures exceed that of seeing one's Ph.D. students do well and succeed. This is one of the unique rewards of academic life. So, it is a great pleasure to serve as an editor of the focus section honoring a former student and friend, Yinsheng Wang, whose achievements have gained him the 2013 Biemann Medal from the ASMS, for "innovative application of mass spectrometry in the field of modified nucleic acids."

Yinsheng has been recognized not only with the Biemann Medal but also with the inaugural Chemical Research in Toxicology Young Investigator Award, for "applying innovative chemical technologies to the solution of important toxicological problems," co-sponsored by the Division of Chemical Toxicology of the American Chemical Society and the ACS journal Chemical Research in Toxicology, 2012. Furthermore, he is a Fellow of the American Association for the Advancement of Sciences (2012-present), for "distinguished contributions to the field of bioanalytical chemistry, particularly in the development of novel analytical methods enabling understanding of the biological consequences of DNA damage." Yinsheng also won a Research Award of the ASMS in 2005. These awards are early and predictive of what is to come in a career that is certain to continue to be productive and of high impact.

Yinsheng came to Washington University in St. Louis in 1997 and chose to work with John-Stephen Taylor and me. John works on photochemical damage to DNA caused by exposure to UV radiation. My role was to assist Yinsheng to learn the fundamentals of mass spectrometry and chemical analysis as could be applied to the structure determination of modified nucleic acids. At the time, this was an active area in my laboratory, where we investigated mechanism of fragmentation of nucleic acids and collaborated to apply MS methods to examine modified nucleic acids that had implications in carcinogenesis.

In four years at Washington University, Yinsheng mastered two fields - mass spectrometry and DNA photochemistry. He wasn't ever deterred by a research challenge. For example, when it became clear that molecular orbital calculations would be useful, he worked with Peter Gaspar in our department to design calculations that complement experimental findings. He took all problems, minor setbacks, and difficulties "in stride" because his top priority was to do science. He worked effectively, independently, and produc-

Correspondence to: Michael Gross; e-mail: mgross@wustl.edu

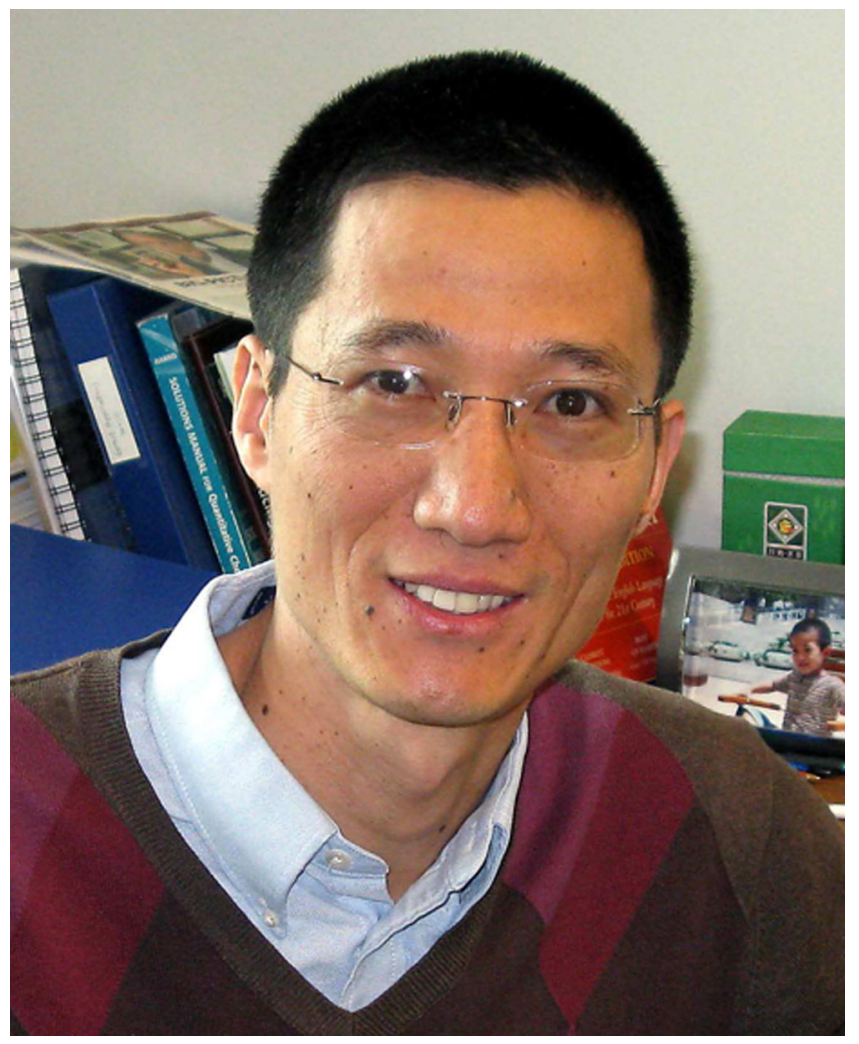

tively, completing nine research papers, in eight of which he is first author, and one book chapter. As John Taylor pointed out at Yinsheng's thesis defense, “a joint Ph.D. student often means that the person is never in either lab; in the case of Yinsheng, he seemed to be simultaneously in both."

When Yinsheng finished his Ph.D., he was clearly focused on an academic career. I recommended he spend a year or two as a postdoctoral, but he wanted to get his career started. He already had an MS from the Chinese Academy of Sciences in another area, had spent a year at SUNY in the US, and had worked in two areas for his Ph.D. He felt that he already had obtained sufficient breadth to begin his independent career, and although I was unsure of his plan, his subsequent career accomplishments clearly show that I was wrong and he was correct.

Starting in 2001 as assistant professor at the University of California-Riverside, Yinsheng has compiled a remarkable record. He rapidly rose through the ranks to become associate professor in 2005 and professor in 2008. He also serves as director of the Environmental Toxicology Graduate Program at Riverside, which he helped organize. During his 
time at Riverside, he has published over 140 articles in peerreviewed journals and four book chapters. In an amazing testimony to the support and respect he receives from his peers, he currently holds three NIH R01 grants, where he is the PI, and one instrument grant. He also enjoys support from an NIH program project grant and another NIH multiPI grant. Another measure of his impact and success is the $19 \mathrm{Ph} . \mathrm{D}$. and 10 postdoctoral students who completed their studies with him. His current group consists of 14 Ph.D. students and four postdoctoral fellows, indicating his productivity and impact are accelerating.

The announcement of the Biemann Medal contained the following description:

Dr. Yinsheng Wang has focused his research on discovering the biological consequences of DNA damage and on unraveling mechanisms of action for anti-tumor drugs and environmental toxicants. His laboratory's use and development of mass spectrometry, synthetic organic chemistry, biochemistry, and molecular biology enables us to understand and quantify, at the molecular level, how various DNA damage products are repaired, and how they perturb the efficient flow and fidelity of genetic information during DNA replication and transcription. Professor Wang has identified and characterized new DNA lesions, including bulky lesions induced by reactive oxygen species. His laboratory developed LC-MS/MS combined with a plasmid-based shuttle vector to quantitatively assess how structurally defined DNA lesions alter the frequency and efficiency of DNA replication and transcription in cells, and to measure the types and frequencies of mutations induced by lesions. They also discovered that $N^{2}$-(1-carboxyethyl)-2'deoxyguanosine is the major stable DNA adduct derived from methylglyoxal, and demonstrated that it is the previously unknown endogenous substrate for DinB (polymerase IV). Dr. Wang's new methods have provided some long-sought biomarkers for oxidative stress: cyclopurine lesions including 8,5'-cyclo-2'-deoxyadenosine and 8,5'-cyclo-2'-deoxyguanosine.

To honor Yinsheng and recognize his award, we have assembled a focus of seven articles from friends and colleagues who also work in the area of mass spectrom- etry and nucleic acid chemistry, and from Yinsheng and his group. The articles provide a snapshot of the role of mass spectrometry in this important area of biochemistry and biomedicine. They are presented in small groups that represent instrumentation, modified nucleic acid characterization, nucleic acid conformation studies, and oligodeoxynucleotide fragmentation.

1) Understanding gas phase modifier interactions in rapid analysis by differential mobility-tandem mass spectrometry by Amol Kafle, Stephen L. Coy, Bryan M. Wong, Albert J. Fornace Jr., James J. Glick, and Paul Vouros

2) Metabolic de-isotoping for improved LC-MS characterization of modified RNAs by Collin Wetzel, Siwei Li, and Patrick A. Limbach

3) Development of a nanoLC/ESI ${ }^{+} \mathrm{HRMS}^{3}$ method for $N-7$ (1-hydroxy-3-buten-2-yl)-guanine DNA adducts of 1,3butadiene in vivo by Dewakar Sangaraju, Peter W. Villalta, Susith Wickramaratne, James Swenberg, and Natalia Tretyakova

4) Structure-specific ribonucleases for MS-based elucidation of higher-order RNA structure by M. Scalabrin, Y. Siu, P. N. Asare-Okai, and Daniele Fabris

5) Native electrospray mass spectrometry of DNA Gquadruplexes in potassium solution by Adrien Marchand and Valerie Gabelica

6) More than charged base loss - revisiting the fragmentation of highly charged oligonucleotides by Adrien Nyakas, Rahel P. Eberle, Silvan R. Stucki, and Stefan Schürch

7) Fragmentation of electrospray-produced deprotonated ions of oligodeoxyribonucleotides containing an alkylated or oxidized thymidine by Pengcheng Wang, Renee T. Williams, Candace R. Guerrero, Debin Ji, and Yinsheng Wang

The editors at JASMS send hearty congratulations to Yinsheng on this much-deserved award, and we invite you, the readers, to celebrate the award by enjoying the seven articles that comprise the focus.

\author{
Michael L. Gross \\ Editor, JASMS \\ Department of Chemistry \\ Washington University in St. Louis \\ St. Louis, MO, USA \\ e-mail:mgross@wustl.edu
}

